\title{
Short-Wave Near-Infrared Spectroscopy of Milk Powder for Brand Identification and Component Analysis
}

\author{
D. Wu, S. Feng, and Y. He ${ }^{1}$ \\ College of Biosystems Engineering and Food Science, Zhejiang University, Hangzhou 310029, China
}

\begin{abstract}
The aim of the present paper was to provide new insight into the short-wave near-infrared (NIR) spectroscopic analysis of milk powder. Near-infrared spectra in the 800- to $1,025-\mathrm{nm}$ region of 350 samples were analyzed to determine the brands and quality of milk powders. Brand identification was done by a least squares support vector machine (LS-SVM) model coupled with fast fixed-point independent component analysis (ICA). The correct answer rate of the ICA-LS-SVM model reached as high as $98 \%$, which was better than that of the LS-SVM (95\%). Contents of fat, protein, and carbohydrate were determined by the LS-SVM and ICA-LS-SVM models. Both processes offered good determination performance for analyzing the main components in milk powder based on short-wave NIR spectra. The coefficients of determination for prediction and root mean square error of prediction of ICA-LS-SVM were $0.983,0.231$, and 0.982 , and $0.161,0.980$, and 0.410 , respectively, for the 3 components. However, there were less than 10 input variables in the ICA-LSSVM model compared with 225 in the LS-SVM model. Thus, the processing time was much shorter and the model was simpler. The results presented in this paper demonstrate that the short-wave NIR region is promising for fast and reliable determination of the brand and main components in milk powder.
\end{abstract}

Key words: short-wave near-infrared spectroscopy, milk powder, support vector machine, component analysis

\section{INTRODUCTION}

Milk powder is a popular nutritional food. The proportions of nutritional components are determined according to the needs of different people, and the nutritional components greatly affect the market price. Recently, consumption of milk powder has increased, and

Received August 6, 2007.

Accepted November 26, 2007.

${ }^{1}$ Corresponding author: yhe@zju.edu.cn with it, some fraudulent phenomena have increased as well. To make enormous profits, some underground factories produce inferior milk powders that contain insufficient or superfluous nutritional contents. Others mix brands of milk powder of different qualities, such as by packing a milk powder belonging to a common brand in the packaging of a high-grade brand. These behaviors badly infringe on the rights and interests of consumers and have caused many tragedies. For example, in 2004, an inferior milk powder incident in Fuyang (Anhui province, China) caused malnutrition in 217 babies, and 12 infants died. In 2006, the protein contents of 3 varieties of Orient brand milk powder (Inner Mongolia province) were found to be only $0.29,0.27$, and $0.42 \%$, respectively, even though the labels on the packaging claimed that the protein contents were between 12 and $16 \%$. These tragedies could be avoided if a fast and accurate analytical method were in place to determine the brands and quality of milk powders. Consumers commonly judge the quality of a milk powder by touch, color, smell, and taste, and by rating its solubility, among other factors. Although these methods are easy, as a primary and experiential method they are subjective and less accurate. A chemical composition analysis and a physical property assessment are routinely performed in the commercial trading of milk powder. However, these methods are time-consuming, destructive, and costly, and they require professional laboratory operations. Therefore, the need exists for a rapid, nondestructive, and less expensive method that is suitable for screening milk powder, at least for authenticity information such as brand and quality.

Near-infrared spectroscopy (NIRS) has been widely applied as a fast and nondestructive analytical method. Near-infrared spectroscopy offers speed of analysis and does not require sample preparation. The near-infrared (NIR) bands mainly correspond to the $\mathrm{C}-\mathrm{H}, \mathrm{O}-\mathrm{H}$, and $\mathrm{N}-\mathrm{H}$ vibrations, which originate from fundamental bands in the midinfrared region. The potential of NIRS has been proved in many papers that have reported on the content of milk powders (Borin et al., 2006; Wu et al., 2007, 2008). Almost all the NIR experiments on 
milk powder have been done in the 1,100 - to $2,500-\mathrm{nm}$ region. However, the short-wave NIR region should also be considered for a quantitative and qualitative analysis of milk powder.

The short-wave NIRS technique (wavelengths ranging from 700 to $1,100 \mathrm{~nm}$ ) has gained interest because the short-wave region is suitable for nondestructive analyses of biological and biomedical materials. The transmittance of light in the 700- to $1,100-\mathrm{nm}$ region is high, so this region is available for designing an excellent detector. It could then be possible for online sensors based on short-wave NIR to be constructed for the nondestructive determination of various components in biological and biomedical materials. The short-wave NIR region has several advantages; for example, the signal exploitation is reliable, the measurement time is low (Bittner et al., 1995), and the effect of intense water bands in the NIR region can be diminished (Reeves, 1994). Sasic and Ozaki (2001) used short-wave NIRS for a quantitative analysis of fat, protein, and lactose in raw milk. He et al. (2007) measured the sugar content of yogurt by using short-wave NIRS. Subedi et al. (2007) investigated the eating quality of mangoes at harvest by using short-wave NIRS.

Because milk powder is a highly complex matrix, its corresponding short-wave NIR data contain highly overlapped peaks, regardless of bandwidth. The results have much noise, and other unrelated information arises from overtones and combinations of such vibrations, rendering them more difficult to interpret (Reid et al., 2005). Thus, it is necessary to use chemometric methods to extract the relevant quantitative information. Although the establishment of a chemometric model may be time-consuming, once the model is established, analysis can be performed within a short time (Brás et al., 2005).

The purpose of the present study was to explore the potential of short-wave NIRS as a rapid and low-cost technique to screen milk powders for characteristic information such as brand and quality of the main components. Short-wave NIR spectra in the 800- to 1,025-nm region of milk powder have been investigated as an important example. A nonlinear mathematical method for the rapid, nondestructive identification of milk powder brands was developed by fast fixed-point independent components analysis (FastICA) and a least squares support vector machine (LS-SVM). In addition, quantitative analyses of fat, protein, and carbohydrate were done based on FastICA and LS-SVM. Quantitative analyses of the main components in milk powder could be used for determination of the contents in milk powder and would be helpful to explain why the different brands of milk powder can be identified by shortwave NIR.

\section{MATERIALS AND METHODS}

\section{Sample Preparation}

Seven brands of 6- to 12-mo infant milk powder were purchased from several local supermarkets. The 7 brands were Wyeth, Frisomel, Yashili, MeadJohnson, Yili, Syrutra, and Beingmate. Each brand included 50 samples from 5 different production dates. Milk powder was stored in an ice-filled cooler and was transported to the laboratory and stored at a low temperature (4 \pm $\left.1^{\circ} \mathrm{C}\right)$. All experiments were conducted at ambient temperature $\left(18\right.$ to $\left.20^{\circ} \mathrm{C}\right)$. Each sample of the milk powder was poured into uniform glass containers $(65 \mathrm{~mm}$ diameter, $14 \mathrm{~mm}$ in height).

\section{Spectral Measurements}

Spectral measurements were taken by using a handheld FieldSpec Pro FR (325 to 1,075 nm)/A110070 (trademarks of Analytical Spectral Devices Inc., Boulder, CO). Near-infrared reflectance spectra in the 700to $1,075-\mathrm{nm}$ region were recorded. To exploit the 10 degree field of view of the probe, the spectroradiometer was placed at a distance of approximately $150 \mathrm{~mm}$ and at a 45-degree angle away from the measurement area. As the only illumination, a Lowell pro-lam 14.5-V Bulb/ 128690 tungsten light source (Ushio Lighting Inc., Osaka, Japan) was used. The halogen lamp could offer a constant light source and covered the entire NIR region. The lamp was placed approximately $300 \mathrm{~mm}$ away from the measurement area and at 45 degrees from the horizontal plane. Because the spectroradiometer measures only the intensity of a light field through a given point in space, short-wave NIR reflectance was computed with spectral measurements both from samples and from a white standard panel with approximately $100 \%$ reflectance from the visible to the short-wave NIR region. The reflectance spectrum of the panel was taken before beginning the spectral scanning experiment. The spectrum of each sample was the average of 30 successive scans. To avoid a low signal-to-noise ratio, only the wavelength region of 800 to $1,025 \mathrm{~nm}$ was used for the calculations. Absorbance data were stored as log $1 / R$ (where $R$ is reflectance) at $1-n m$ intervals (225 spectral data points). These 30 values were then averaged and stored as the absorbance value of this sample. All spectral data were stored in a computer and processed by using $\mathrm{RS}^{3}$ software for Windows (Analytical Spectral Devices Inc.) designed with a graphical user interface.

\section{Content Measurement of the Main Components}

Fat content was measured by the Röse-Gottlieb method following GB/T 5413.3-1997 (National Stan- 
dard of P. R. China). The fat content value was the weight of fat per $100 \mathrm{~g}$ of milk powder. Protein content was determined by the Kjeldahl method as described by GB/T 5413.1-1997 (National Standard of P. R. China), and the factor 6.38 was used to convert the nitrogen values to protein. The protein content value was the weight of protein per $100 \mathrm{~g}$ of milk powder. Carbohydrate content was determined by the method of Lane and Eynor (AOAC, 1984) as described by GB/ T 5413.5-1997 (National Standard of P. R. China). The carbohydrate content value was the sum of the weights of sucrose and lactose per $100 \mathrm{~g}$ of milk powder.

\section{Chemometrics of LS-SVM}

The SVM is a promising method proposed by Vapnik (1998) and is processed based on statistical learning theory. The structural risk minimization principle (SRM) is embodied instead of the traditional empirical risk minimization principle, which is used by conventional neural networks to avoid overfitting and multidimensional problems. The LS-SVM is an optimized algorithm based on the standard SVM by Suykens et al. (2002). The LS-SVM has the capability for linear and nonlinear multivariate calibration and solves the multivariate calibration problems in a relatively fast way (Suykens and Vanderwalle, 1999). It uses a linear set of equations instead of a quadratic programming problem to obtain the support vectors (Li et al., 2007). It adopts a least squares linear system as the loss function and is applied in the pattern recognition and nonlinear evaluation. It is capable of learning in a high-dimensional feature space with fewer training data.

In the LS-SVM algorithm, a nonlinear mapping function $\phi(\cdot)$ is applied for constructing the regression model, whereas the input data are mapped to a higher dimensional feature space. When the least squares support vector is used as a soft testing tool, a new optimization problem is formulated in the case of SRM. The Lagrange function is then adopted to solve this optimization problem. Based on Mercer's theory, there is an equation between the kernel function $K\left(x_{i}, x_{j}\right)$ and the mapping function $\phi(\cdot)$ :

$$
\phi\left(x_{k}\right)^{T} \phi\left(x_{l}\right)=K\left(x_{k}, x_{l}\right), k, l=1, \ldots, N .
$$

Common examples of the kernel function contain a linear, polynomial, radial basis function (RBF) kernel and a multilayer perceptron. In our work, the RBF kernel was selected. Finally, the LS-SVM regression model could be obtained as

$$
y(x)=\sum_{k=1}^{N} \alpha_{k} K\left(x, x_{k}\right)+b .
$$

In the SVM or LS-SVM process, the proper kernel function and the best kernel parameters need to be determined. However, no systematic methodology is available for prior selection of the kernel function. The $\mathrm{RBF}$ kernel is a nonlinear function and a more compacted supported kernel. The RBF kernel can reduce the computational complexity of the training procedure while giving good performance under general smoothness assumptions; hence, the RBF kernel was used in this paper. A good LS-SVM classification model with high prediction accuracy and stability was built, based on the proper parameter setting. A grid-search technique was applied to find the optimal parameter values; these included the regularization parameter gam $(\gamma)$ and the RBF kernel function parameter sig2 $\left(\sigma^{2}\right)$, which is the bandwidth in the common case of the RBF kernel. The parameter $\gamma$ determines the trade-off between SRM and ERM, and is important to improve the generalization performance of the LS-SVM model. The parameter $\sigma^{2}$ controls the value of the function classification error and influences the number of initial eigenvalues and eigenvectors directly. A small value of $\sigma^{2}$ can lead to overfitting, whereas a large value of $\sigma^{2}$ will make the model simpler but not accurate. Moreover, $\sigma^{2}$ reflects the sensitivity of the LS-SVM model to noise from the input variables. In this study, these parameters were optimized with values of $\gamma$ in the range of $2^{-1}$ to $2^{10}$ and with values of $\sigma^{2}$ in the range of 2 to $2^{15}$, with adequate increments by the grid-search technique of leave-oneout cross-validation. These ranges were set based on experience in using geometric steps. For each combination of the $\gamma$ and $\sigma^{2}$ parameters, the root mean square error of cross-validation was calculated and the optimal parameters were selected when they produced smaller root mean square errors of cross-validation.

To avoid the effect of differently defined numbers for each class, the algorithm of the LS-SVM classification model allowed only 2 classes to be classified because the class numbers were set as -1 and 1 . When there were more than 2 classes, the LS-SVM classification model needed to encode and decode a multiclass classification task into multiple binary classifiers. The coding was defined by a codebook. The codebook was represented by a matrix in which the columns represented all the different classes and the rows indicated the results of the binary classifiers. The 7 brands with original labels $[1,2,3,4,5,6$, and 7] were encoded in the following codebook (by using minimum output coding; Table 1). Thus, there were 3 levels in each row of binary classifiers in this study, and the LS-SVM classification model needed to process these 3 rows (3 dimensions) separately. After the classification process, the classified results of LS-SVM were decoded again to their original form. 
Table 1. Multiple binary classifiers for the input set of the least squares support vector machine for 7 brands of milk powder

\begin{tabular}{lrrrrrrr}
\hline & \multicolumn{7}{c}{ Brand no. } \\
\cline { 2 - 8 } Item & 1 & 2 & 3 & 4 & 5 & 6 & 7 \\
\hline Binary classifier & -1 & -1 & -1 & -1 & 1 & 1 & 1 \\
& -1 & -1 & 1 & 1 & -1 & -1 & 1 \\
& -1 & 1 & -1 & 1 & -1 & 1 & -1 \\
\hline
\end{tabular}

All the aforementioned calculations were performed by using MATLAB 7.0 (The Math Works Inc., Natick, MA). The free LS-SVM toolbox (LS-SVM version 1.5, Suykens, Leuven, Belgium) was applied with MATLAB to derive all the LS-SVM models.

\section{Chemometrics of FastICA}

The number of absorbance values was 225 for each sample from 800 to $1,025 \mathrm{~nm}$, so there were $225 \mathrm{ab}-$ sorbance variables. Although these variables could be used to establish the classification model by LS-SVM, to improve the training speed and reduce the calculation complexity and training error, the FastICA process was applied to enhance the brand features and reduce the data dimensionality. Independent components analysis (ICA) was previously developed as a well-established statistical signal-processing technique (Singer, 2006). Blind source separation (the cocktail party problem) and feature extraction are 2 interesting applications of ICA. Independent components analysis can effectively decompose a set of measured mixed signals into the independent components (IC) with a minimal loss of information and without any additional information about the signal origin. FastICA is a computationally highly efficient ICA developed by Hyvärinen and Oja (1997). FastICA is considered a fixed-point algorithm for maximum likelihood estimation of the ICA data model. It is parallel, distributed, computationally simple, and requires little memory space (Hyvärinen and Oja, 2000).

As developed by Hyvärinen and Oja (2000), the process in short is:

1. Choose an initial random weight vector $w(0)$ and let $k=1$.

2. Let $w(k)=E\left\{x g\left(w(k-1)^{T} x\right)\right\}-E\left\{g^{\prime}\left(w(k-1)^{T} x\right)\right\} w(k$ -1 ), where $g$ is the first-derivative of the function G. The expectations can be estimated by using a large sample of $x$ vectors by computing over successive points.

3. Let $w(k)=w(k) /\|w(k)\|$.

4. If $\left|w(k)^{T} w(k-1)\right|$ is not close enough to 1 , let $k=k$ +1 and go back to step 2 . Otherwise, output the vector $w(k)$.

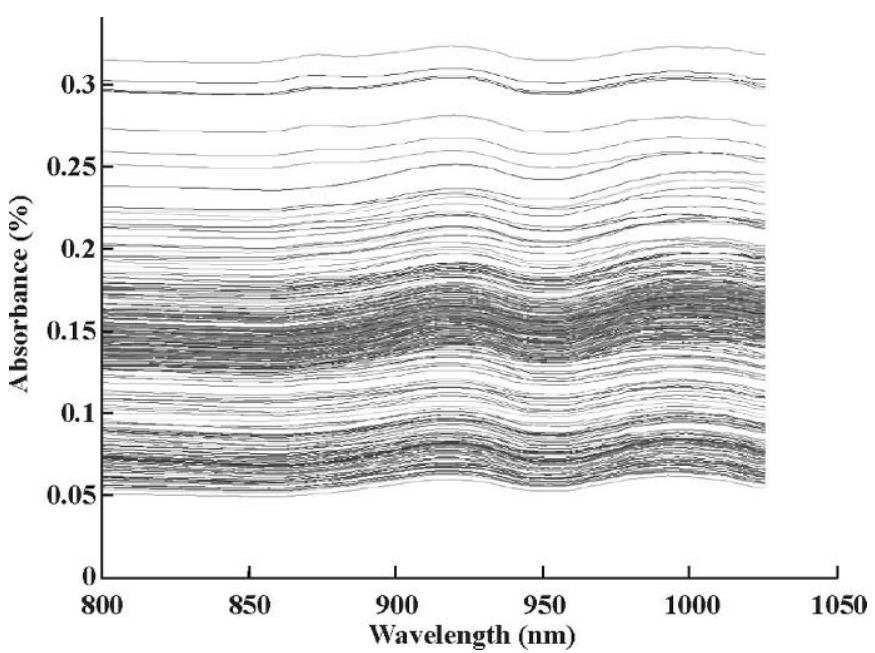

Figure 1. Original short-wave near-infrared spectra of milk powder.

In this study, the FastICA process was carried out in MATLAB 7.0 on spectral data of all the samples from wavelengths 800 to $1,025 \mathrm{~nm}$. The parameter setting was as follows: the number of signals was 225 , the reduced dimension was set at 15 , the number of IC was 15 , the stopping criterion was 0.0001 , and the function of nonlinearity was Gaussian $\left[\mathrm{g}(\mathrm{u})=\mathrm{u} \times \exp \left(-\mathrm{u}^{2} / 2\right)\right]$. Independent components were obtained through FastICA and then set as the optimal input feature subset for LS-SVM.

\section{Model Evaluation}

The predictive performance of the model was evaluated by the following standards. When it was for brand identification, the correct answer rate (CAR, \%) was used. When it was for the main quality component analysis, several standards were used, such as the coefficients of determination for calibration $\left(\mathrm{R}_{\mathrm{c}}^{2}\right)$ and prediction $\left(R_{p}^{2}\right)$, the root mean square error of calibration and the root mean square error of prediction (RMSEP), and the residual predictive deviation (RPD). Some other standards, such as slope and bias, could be taken into consideration for distinguishing systematic errors and studying the correlation between the reference and short-wave NIR models. A good model should have a high correlation coefficients of determination and RPD values coupled with low values for the root mean square error of calibration and RMSEP.

\section{RESULTS AND DISCUSSION}

\section{Features of Short-Wave NIR Spectra}

The absorbance spectra of milk powder from 800 to $1,025 \mathrm{~nm}$ are shown in Figure 1. Compared with the 
Table 2. First 4 principal components and cumulative reliabilities

\begin{tabular}{lcccc}
\hline & \multicolumn{4}{c}{ Principal component } \\
\cline { 2 - 5 } Item & 1 & 2 & 3 & 4 \\
\hline Reliability & 80.35913 & 10.76352 & 5.917838 & 1.809022 \\
Cumulative reliability (\%) & 80.35913 & 91.12265 & 97.04049 & 98.84951 \\
\hline
\end{tabular}

NIR spectra of raw milk in the 800- to 1,025 -nm region (Sasic and Ozaki, 2001), there was not a broad feature around $970 \mathrm{~nm}$ because of the $2 \mathrm{v} 1+\mathrm{v} 3$ ( 1 1, symmetric stretching; v3, asymmetric stretching) vibration of water (Osborne and Fearn, 1986) and because the main moisture was evaporated from the milk during the process of manufacturing the milk powder. The baseline changes were the main differences from one spectrum to another, whereas they were unavoidable in the pure milk powder spectra. In the milk powder spectra, the baseline changes may have been induced by light scattering because of the size of the powder granules. Different types of pretreatments have been applied to the spectra to eliminate them (Chen et al., 1999; Tsenkova et al., 1999). One of the pretreatment methods often used is the standard normal variate (SNV). The SNV was applied for light-scattering correction and to reduce the changes in the light path length (Barnes et al., 1989). Hence, we applied SNV to the spectra. The pretreatments were implemented by The Unscrambler version 9.6 software (Camo Process AS, Oslo, Norway). Because the absorbance curves of all the samples overlapped, it was difficult to distinguish the different brands. Thus, it was not feasible to analyze the spectral figure directly, and some chemometric methods were needed to mine the potential information.

\section{Clusters of the Principal Component Analysis}

After the SNV spectral pretreatment, a principal components analysis was carried out by The Unscrambler version 9.6 software (Camo Process AS), and principal components were obtained. Table 2 shows the cumulative reliabilities of the first 4 principal components. Results indicated that the first 3 principal components could explain $97.04 \%$ of the total variance. The fourth principal component contributed only an additional $1.81 \%$. Hence, the first 3 principal components could represent most of the feature information of the original spectra. Figure 2 shows the score plot of the 7 varieties by using the first 3 principal components. The score plot represents clusters of different brands. Samples were well distributed in the 3-dimensional score space except for samples of brands 5 and 7 . One sample of brand 7 was mixed in the clustering of brand 5 . The border between brands 5 and 7 was not distinct. The

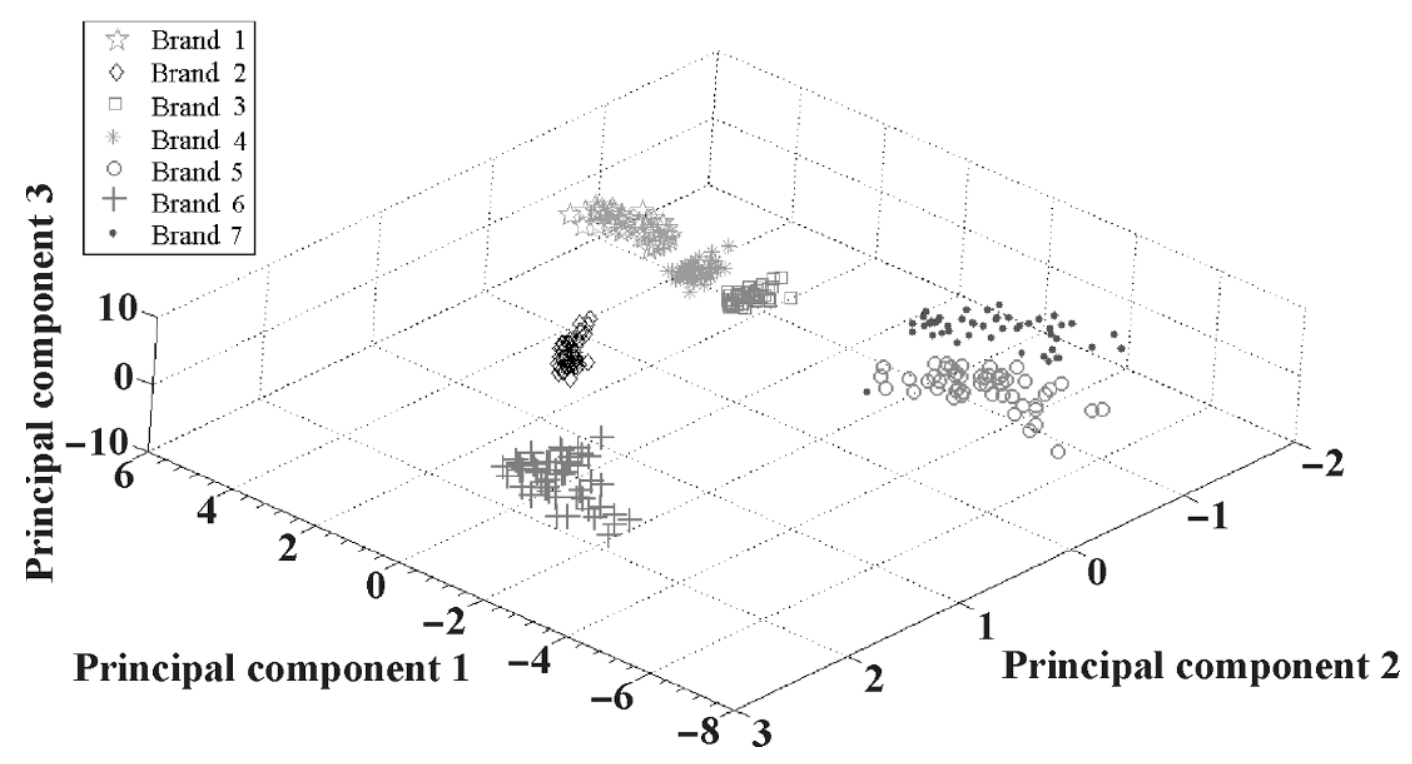

Figure 2. Score plot for first 3 principal components (401 to 1,000 nm) of 7 brands of milk powder. 
Table 3. Performance of the least squares support vector machine for brand identification of milk powders

\begin{tabular}{lccrcccccc}
\hline & & & & & & & & & \multicolumn{2}{c}{$\begin{array}{c}\text { Correct answer } \\
\text { rate (\%) of the } \\
\text { prediction set }\end{array}$} \\
\hline 1 & 234 & 0.5737 & 3.9281 & 5.0649 & 37.9511 & 64.0647 & 21.5739 & 94.8497854 \\
2 & 234 & 1.1828 & 53.0651 & 2.1815 & 13.886 & 65.9265 & 64.4914 & 96.1538462 \\
\hline
\end{tabular}

cluster plot suggested that the different brands of milk powder could be distinguished by using short-wave NIR spectra.

\section{LS-SVM Classification Model}

For the quantitative analysis, 2 models were developed by using different sample sets consisting of 116 spectra each. On the basis of the models developed, the LS-SVM was applied to predict the 234 remaining samples after SNV pretreatment, similar to the calibration pretreatment. The prediction results for these 2 separate models are shown in Table 3.

Results revealed that the CAR of the 2 models improved to approximately 95\%. A satisfying prediction precision was achieved. Even 2 brands with many closed component contents, such as brands 6 and 7, could be identified well by short-wave NIR. For example, in model 1, only 1 sample of brand 6 (of 34 samples for prediction) was identified as brand 7 and the others were identified correctly. All the samples of brand 7 (of 33 samples for prediction) were identified correctly. The most identification errors were found between brands 5 and 7. As one can see in Figure 3, some spectra from brands 5 and 7 had obvious peaks around $875 \mathrm{~nm}$, whereas in Figure 4, there are no such features of other brands. From the spectral analysis of the peaks around $875 \mathrm{~nm}$ and the erroneously identified samples belonging to brands 5 and 7 , we found that the most falsely identified samples had such peaks. Samples without such peaks were identified correctly. However, this feature was not obvious in the original spectra (Figure 1). This might be because the SNV pretreatment enlarged the differences at $875 \mathrm{~nm}$. The amplified noise caused the false identification of these samples.

\section{FastICA and ICA-LS-SVM Processes}

The top 15 IC obtained through FastICA contained more than $99 \%$ of the information from the original spectral data. The models analyzed here were the same as before. The 116 samples of the calibration set were used to establish the ICA-LS-SVM model, and the remaining 234 samples were used for prediction. Classified results of the prediction set of the LS-SVM models were obtained based on different numbers of IC. Table
4 shows prediction results of the LS-SVM models based on different numbers of IC. The best CAR of both models based on the optimal IC number were higher than that of the LS-SVM model based on 225 absorbance variables, and the processing time was shorter. From Table 4 , one can see that the CAR were directly proportional to the IC numbers except for those of the 15 IC of the prediction set in model 2 . This may be because the 15 IC here not only had useful information for brand identification, but also included some noise, which was bad for identification. The LS-SVM model with a small number of IC could not obtain a good classification result, although a small IC number meant less calculation time. Because no systematic methodology is available for choosing the best number of IC, the determination of number needed to be compared with the final classification results based on LS-SVM models with different numbers of IC. Twelve IC would be suggested here in the ICA-LS-SVM model.

Upon comparing the 2 chemometric models, the performance of the ICA-LS-SVM was found to be better than that of the LS-SVM. We concluded that the LSSVM is a powerful classification tool for spectral analysis and that the ICA is a good tool for quick convergence and could reduce computation well.

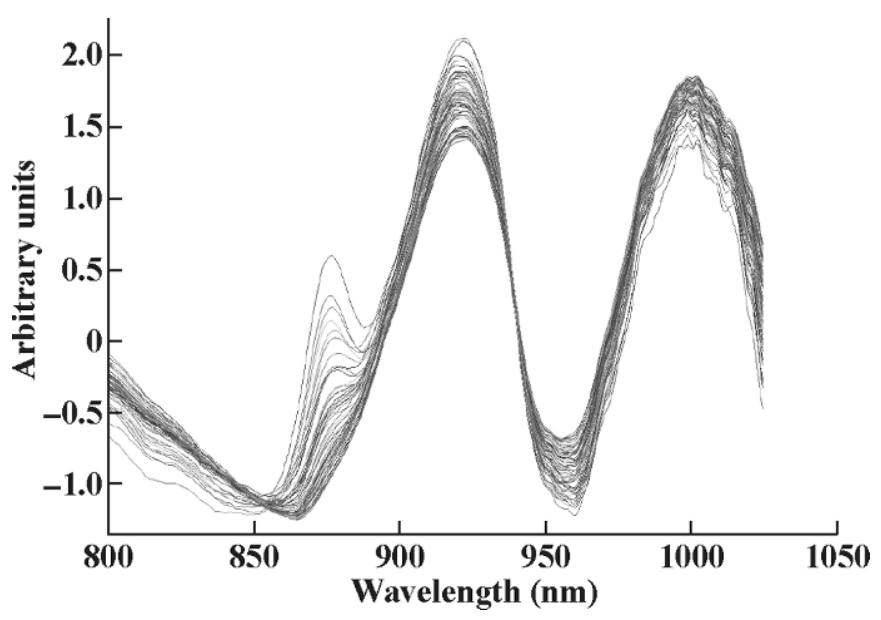

Figure 3. Short-wave near-infrared spectra of brands 5 and 7 after the standard normal variate pretreatment. 


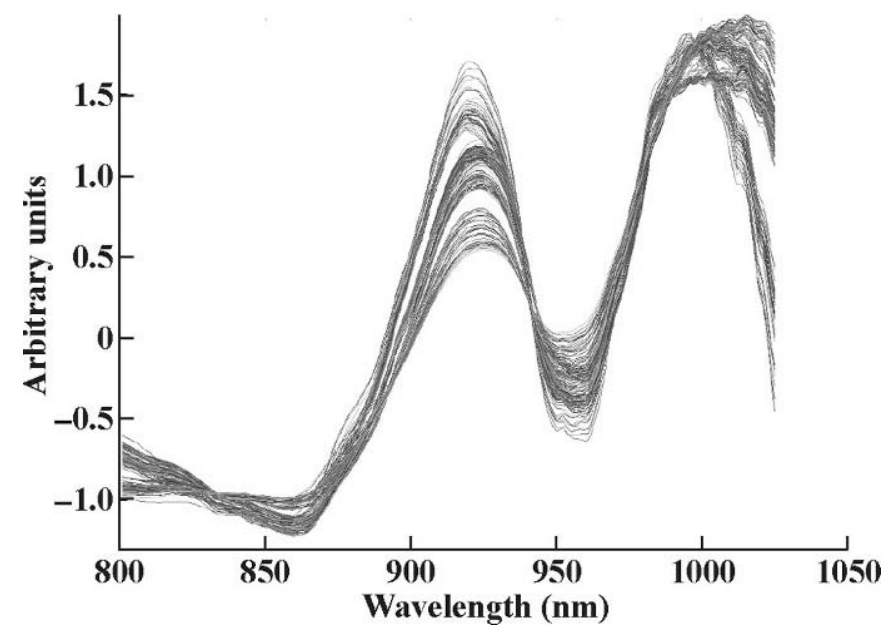

Figure 4. Short-wave NIR spectra of brands 1 to 4 and 6 after the standard normal variate pretreatment.

\section{Generalization Ability of the ICA-LS-SVM Model}

A study of the different sample numbers for the calibration and prediction sets was executed to further investigate the generalization ability of the ICA-LS-SVM. The models analyzed here were the same as before. The CAR of all validations were $100 \%$. One can see in Table 5 that the variation in CAR of each model was not large except for that of model 1, with 25 samples for calibration and the other 325 samples for prediction. The reason may be that the 25 samples for calibration was a small number and did not contain enough feature

Table 4. Prediction results of the least squares support vector machine model based on different numbers of independent components

\begin{tabular}{lcccc}
\hline Model & Set & Number & $\begin{array}{c}\text { Independent } \\
\text { components }\end{array}$ & $\begin{array}{c}\text { Correct answer } \\
\text { rate }(\%) \text { of the } \\
\text { prediction set }\end{array}$ \\
\hline 1 & Calibration & \multirow{2}{*}{116} & 3 & 61.54 \\
& & 6 & 90.60 \\
& & 9 & 100 \\
& & 12 & 100 \\
& & 15 & 100 \\
& & 3 & 36.91 \\
& & & 6 & 73.39 \\
& & 9 & 93.99 \\
& & & 12 & 97.00 \\
& & & 15 & 97.85 \\
& & & 3 & 46.55 \\
& & & 6 & 95.69 \\
& & 9 & 100 \\
& & & 12 & 100 \\
& & & 15 & 100 \\
& & & 3 & 27.35 \\
& & & 6 & 73.50 \\
& & & 9 & 97.86 \\
& & & &
\end{tabular}

information on each brand. Having 25 samples for calibration meant that there were only 3 or 4 samples for each brand, so if these 3 or 4 samples contained the main feature of their brand, then the CAR would be high, as in model 2 with 25 samples for calibration and the other 325 samples for prediction. In addition, we found that the $\sigma_{2}^{2}$ of model 1 , with 25 samples for calibration, was smaller than the other $\sigma_{2}^{2}$. A small value of $\sigma^{2}$ is known to lead to overfitting. The CAR of calibration of model 1, with 25 samples for calibration, was $100 \%$, whereas the CAR of prediction was decreased to 83\%, and this was obviously overfitting. The main identification error of model 1, with 25 samples for calibration, was that 28 samples for brand 7 (of a total 46 samples) were identified as brand 5 . The code for brand 5 was [ $1-1-1]$ and the one for brand 7 was [ $11-1$ l (Table 1), so the identification of brands 5 and 7 depended on dimension 2 . The small $\sigma_{2}^{2}$ caused overfitting, and finally more than half the samples of brand 7 in model 1, with 25 samples for calibration, were failed for identification.

We concluded that the variation in sample numbers in calibration had less influence on the performance of the ICA-LS-SVM model and that its generalization ability was good. Moreover, the small number of samples for calibration meant that less time was consumed for model establishment. Hence, the ICA-LS-SVM could be used effectively for application, with a small number of samples used for model establishment. However, the number of samples for calibration should not be too small to affect the identification ability of the established model.

\section{Analysis of the Main Quality Components}

The previous study showed that the milk powder brands could be determined from short-wave NIR spectra, but we needed to explain why there were differences in the spectral measurements from different brands. In this study, the short-wave NIR spectra of the main quality factors for the 7 milk powder brands were studied. Several main components such as fat, proteins, and carbohydrate are important factors that determine the quality of milk powder. Products of different brands contain different qualities of these components. The contents of the main components of milk powder from each brand are shown in Table 6 . The quality of some brands was close. In this study, the short-wave NIR region was considered for the quantitative analysis of fat, proteins, and carbohydrate in milk powder. The component analysis of short-wave NIRS of milk powder can be used for content determination of the main components and would be helpful to explain why spectra of the short-wave NIR region could be used for brand identification of the milk powders. 
Table 5. Prediction results of the independent component analysis-least squares support vector machine models based on different number sets

\begin{tabular}{rccccccccc}
\hline Model & $\begin{array}{c}\text { Calibration } \\
\text { no. }\end{array}$ & $\begin{array}{c}\text { Prediction } \\
\text { no. }\end{array}$ & \multicolumn{1}{c}{$\gamma_{1}$} & \multicolumn{1}{c}{$\gamma_{2}$} & \multicolumn{1}{c}{$\gamma_{3}$} & \multicolumn{1}{c}{$\sigma_{1}^{2}$} & \multicolumn{1}{c}{$\sigma_{2}^{2}$} & $\sigma_{3}^{2}$ & $\begin{array}{c}\text { Correct answer } \\
\text { rate (\%) of the } \\
\text { prediction set }\end{array}$ \\
\hline 1 & 116 & 234 & 0.14 & 0.80 & 152.19 & 16.33 & 51.45 & 33.96 & 97.00 \\
& 87 & 263 & 1.90 & 2.21 & 24.30 & 68.40 & 10.35 & 12.32 & 94.30 \\
& 50 & 300 & 2.69 & 2.18 & 8.82 & 12.67 & 65.45 & 48.48 & 97.69 \\
& 25 & 325 & 4.74 & 2.05 & 76.40 & 16.87 & 5.37 & 16.88 & 83.08 \\
& 116 & 234 & 0.33 & 3.93 & 166.46 & 17.89 & 64.07 & 18.64 & 98.29 \\
& 87 & 263 & 0.85 & 24.83 & 9.19 & 6.87 & 23.40 & 34.35 & 98.86 \\
& 50 & 300 & 1.86 & 4.23 & 17.21 & 8.53 & 10.06 & 72.81 & 95.67 \\
& 25 & 325 & 26.41 & 128.41 & 46.32 & 7.07 & 43.43 & 23.77 & 96.92 \\
\hline
\end{tabular}

Fat Content Analysis. It is well known that fat in milk powder can be satisfactorily predicted from NIR spectra in the 1,100- to 2,500-nm region by use of chemometrics (Barabássy, 2001; Wu et al., 2007). Thus, 2 models were developed using different sample sets consisting of 116 spectra each. The remaining 234 milk powder spectra were used for prediction. The sample set here was the same as before. Two chemometric methods, namely, LS-SVM and ICA-LS-SVM, were applied. Independent components were obtained through FastICA and then set as the optimal input feature subset for LS-SVM. The best performance of higher $\mathrm{R}_{\mathrm{P}}^{2}$ and lower RMSEP was achieved when the number of IC was 8 . The results of the calibration and prediction for these 2 separate models are shown in Table 7. Only small differences existed between the 2 models, which differed slightly in the ranges of fat content variation. Model 2 yielded slightly better results than those of model 1. Both processes performed well. However, there were only 8 input variables in the ICA-LS-SVM model compared with 225 in the LS-SVM model. Thus, the processing time was much shorter and the model was simpler. Residual predictive deviation values showed a reliable ability for fat determination and the higher RPD up to 15 was considered adequate for online application (Williams and Norris, 2001). Thus, very good determination performance for the fat content in milk powder could be achieved by short-wave NIRS.

The short-wave NIR spectral technique was successful in determining the fat content in milk powder. That meant the spectra of the short-wave NIR region had some relationship with the fat content. Figure 5 shows the prediction results of model 2 by the LS-SVM process. One can see that samples of brands 4,5 , and 6 could be identified well because their fat content was different. Samples of brands 2 and 3 were completely mixed, and this was because the fat content of these 2 brands was close. Some samples of brand 7 were mixed with those of brand 1 , so the brands of milk powder with different fat contents could be identified by shortwave NIR spectra. Other components might be helpful for the identification of mixed parts.

Protein Content Analysis. Protein is another important component of milk powder, and it can be pre-

Table 6. Contents of the main components in 7 brands of milk powder ${ }^{1}$

\begin{tabular}{|c|c|c|c|c|c|c|c|}
\hline \multirow[b]{2}{*}{ Component } & \multicolumn{7}{|c|}{ Brand } \\
\hline & 1 & 2 & 3 & 4 & 5 & 6 & 7 \\
\hline \multicolumn{8}{|l|}{ Protein (g) } \\
\hline Maximum & 18.14 & 15.28 & 18.00 & 15.41 & 17.83 & 17.81 & 17.54 \\
\hline Minimum & 17.72 & 14.77 & 17.56 & 14.97 & 17.43 & 17.33 & 17.05 \\
\hline Mean & 17.98 & 14.99 & 17.81 & 15.20 & 17.60 & 17.56 & 17.27 \\
\hline SD & 0.10 & 0.10 & 0.09 & 0.11 & 0.09 & 0.11 & 0.11 \\
\hline \multicolumn{8}{|l|}{ Fat (g) } \\
\hline Maximum & 20.22 & 21.76 & 21.80 & 16.41 & 19.29 & 21.49 & 21.21 \\
\hline Minimum & 19.79 & 21.22 & 21.35 & 15.88 & 18.75 & 20.79 & 20.02 \\
\hline Mean & 20.00 & 21.51 & 21.61 & 16.20 & 18.98 & 21.09 & 20.47 \\
\hline SD & 0.09 & 0.10 & 0.10 & 0.11 & 0.10 & 0.15 & 0.21 \\
\hline \multicolumn{8}{|c|}{ Carbohydrate (g) } \\
\hline Maximum & 56.17 & 57.62 & 51.33 & 60.28 & 56.25 & 53.16 & 54.57 \\
\hline Minimum & 55.82 & 57.04 & 50.66 & 59.82 & 55.63 & 51.47 & 51.84 \\
\hline Mean & 56.00 & 57.33 & 51.00 & 60.02 & 55.98 & 52.46 & 53.31 \\
\hline SD & 0.09 & 0.13 & 0.14 & 0.12 & 0.13 & 0.35 & 0.43 \\
\hline
\end{tabular}

${ }^{1} \mathrm{n}=50$ samples for each brand. 
BRAND AND COMPONENT ANALYSIS OF MILK POWDER BY INFRARED SPECTROSCOPY

Table 7. Results for fat content determination in milk powder from the short-wave near-infrared spectra ${ }^{1}$

\begin{tabular}{lclcccccc}
\hline \multirow{2}{*}{ Set } & Model & \multicolumn{1}{c}{ Method } & $\mathrm{R}_{\mathrm{c}}^{2}$ or $\mathrm{R}_{\mathrm{P}}^{2}$ & $\begin{array}{c}\text { RMSEC } \\
\text { or RMSEP }\end{array}$ & Bias & Slope & Offset & RPD \\
\hline Calibration & \multirow{2}{*}{1} & LS-SVM & 0.996 & 0.100 & $5.64 \mathrm{E}-10$ & 0.992 & 0.144 & 17.96 \\
& & ICA-LS-SVM & 0.997 & 0.096 & $-1.90 \mathrm{E}-06$ & 0.993 & 0.126 & 18.55 \\
& 2 & LS-SVM & 0.996 & 0.112 & $-1.20 \mathrm{E}-09$ & 0.991 & 0.165 & 16.06 \\
\multirow{3}{*}{ Prediction } & \multirow{2}{*}{1} & ICA-LS-SVM & 0.997 & 0.097 & $-1.40 \mathrm{E}-07$ & 0.994 & 0.109 & 18.47 \\
& & LS-SVM & 0.981 & 0.238 & 0.02 & 0.970 & 0.605 & \\
& \multirow{2}{*}{2} & ICA-LS-SVM & 0.975 & 0.273 & -0.01 & 0.967 & 0.655 & \\
& & LS-SVM & 0.982 & 0.232 & -0.02 & 0.996 & 0.048 & \\
& & ICA-LS-SVM & 0.983 & 0.231 & 0.01 & 0.951 & 0.988 & \\
\hline
\end{tabular}

\begin{abstract}
${ }^{1}$ Fat statistics in calibration model 1 : minimum $=15.93 \mathrm{~g}$; maximum $=21.80 \mathrm{~g}$; mean $=19.97 \mathrm{~g} ; \mathrm{SD}=$ $1.79 \mathrm{~g}$. Fat statistics in prediction model 1: minimum $=15.88 \mathrm{~g} ;$ maximum $=21.79 \mathrm{~g} ;$ mean $=19.98 \mathrm{~g} ; \mathrm{SD}=$ 1.76 g. Fat statistics in calibration model 2 : minimum $=15.88$ g; maximum $=21.79$ g; mean $=19.96$ g; $\mathrm{SD}=$ $1.79 \mathrm{~g}$. Fat statistics in prediction model 2 : minimum $=15.93 \mathrm{~g}$; maximum $=21.80 \mathrm{~g}$; mean = $19.98 \mathrm{~g}$; $\mathrm{SD}=1.76 \mathrm{~g} . \mathrm{R}_{\mathrm{c}}^{2}=$ coefficient of determination for calibration; $\mathrm{R}_{\mathrm{p}}^{2}=$ coefficient of determination for prediction; RMSEC $=$ root mean square error of calibration; $\mathrm{RMSEP}=$ root mean square error of prediction; $\mathrm{RPD}=$ residual predictive deviation; LS-SVM = least squares support vector machine; ICA-LS-SVM = independent component analysis-least squares support vector machine.
\end{abstract}

dicted well from NIR spectra in the 1,100- to 2,500$\mathrm{nm}$ region (Barabássy, 2001; Wu et al., 2008). Thus, 2 models were developed by using different sample sets consisting of 116 spectra each. The sample set here was the same as before. Two chemometric methods, namely, LS-SVM and ICA-LS-SVM, were applied. The best performance of the ICA-LASVM model was achieved when the number of IC was 5 . The results of calibration and prediction for these 2 separate models are shown in Table 8. Both processes offered good performance. However, there were only 5 input variables in the ICA-LSSVM model compared with 225 in the LS-SVM model. The RPD values from both methods were also high enough for online application.

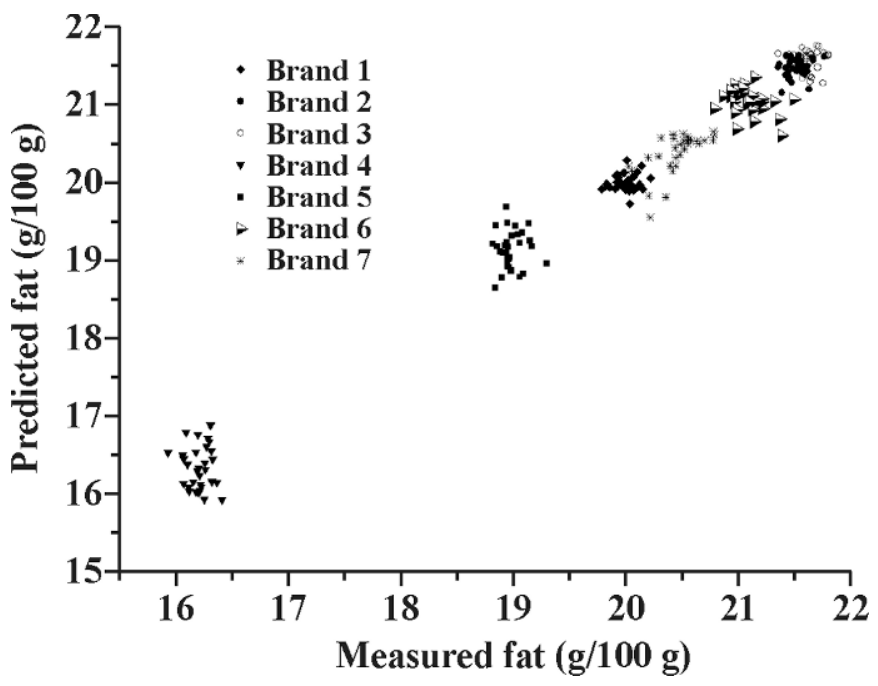

Figure 5. Correlation between predicted and measured values for fat content for the prediction set obtained by the least squares support vector machine process.
Thus, the short-wave NIRS technique was also successful in determining the protein content of milk powder, and there were some relationships between protein content and short-wave NIR spectra. Figure 6 shows the prediction results of model 2 by the LS-SVM process. One can see that although brands 2 and 4 were mixed, they could be distinguished from the others well. Brands 1 and 7 were separated from the others, whereas brands 5 and 6 were mixed. Hence, the brands of milk powder with different protein contents could be identified by the short-wave NIR spectra. Moreover, some samples of brands 5 and 6 were mixed with those of brand 3. Other components might be helpful for identifying the mixed parts.

Carbohydrate Content Analysis. The models analyzed here were the same as before. The results of calibration and prediction for these 2 separate models are shown in Table 9. The best performance of the ICALASVM model was achieved when the number of IC was 6 . Both processes offered good performance. However, there were only 6 input variables in the ICA-LS-SVM model compared with 225 in the LS-SVM model. Thus, very good determination performance for the carbohydrate content in milk powders could be achieved by short-wave NIRS. There was a relationship between carbohydrate content and short-wave NIR spectra. In addition, the RPD values of the LS-SVM models were in the hundreds, which were too high and were presumed to be overfitting.

Figure 7 shows the prediction results of model 2 by the LS-SVM process. One can see that brands 2,3 , and 4 could be identified well according to their different carbohydrate contents, whereas brands 1 and 5 were completely mixed. This was because the carbohydrate contents of these 2 brands were close. Some samples of 
WU ET AL.

Table 8. Results for protein determination in milk powder from the short-wave near-infrared spectra ${ }^{1}$

\begin{tabular}{|c|c|c|c|c|c|c|c|c|}
\hline Set & Model & Method & $\mathrm{R}_{\mathrm{c}}^{2}$ or $\mathrm{R}_{\mathrm{P}}^{2}$ & $\begin{array}{c}\text { RMSEC } \\
\text { or RMSEP }\end{array}$ & Bias & Slope & Offset & $\mathrm{RPD}$ \\
\hline \multirow[t]{4}{*}{ Calibration } & \multirow[t]{2}{*}{1} & LS-SVM & 0.997 & 0.069 & $2.11 \mathrm{E}-09$ & 0.994 & 0.100 & 17.27 \\
\hline & & ICA-LS-SVM & 0.993 & 0.093 & $3.75 \mathrm{E}-08$ & 0.990 & 0.156 & 12.70 \\
\hline & \multirow[t]{2}{*}{2} & LS-SVM & 0.996 & 0.060 & $-2.26 \mathrm{E}-08$ & 0.992 & 0.125 & 19.83 \\
\hline & & ICA-LS-SVM & 0.994 & 0.084 & $-3.9 \mathrm{E}-10$ & 0.991 & 0.150 & 14.10 \\
\hline \multirow[t]{4}{*}{ Prediction } & \multirow[t]{2}{*}{1} & LS-SVM & 0.982 & 0.165 & -0.04 & 1.017 & -0.309 & \\
\hline & & ICA-LS-SVM & 0.975 & 0.187 & -0.03 & 0.976 & 0.369 & \\
\hline & \multirow[t]{2}{*}{2} & LS-SVM & 0.981 & 0.231 & 0.02 & 1.010 & -0.133 & \\
\hline & & ICA-LS-SVM & 0.982 & 0.161 & -0.06 & 0.956 & 0.757 & \\
\hline
\end{tabular}

\footnotetext{
${ }^{1}$ Protein statistics in calibration model 1: minimum $=14.77 \mathrm{~g}$; maximum $=18.16 \mathrm{~g}$; mean $=16.90 \mathrm{~g} ; \mathrm{SD}=$ $1.19 \mathrm{~g}$. Protein statistics in prediction model 1: minimum = $14.85 \mathrm{~g}$; maximum = $18.14 \mathrm{~g}$; mean = $16.93 \mathrm{~g}$; $\mathrm{SD}=1.18 \mathrm{~g}$. Protein statistics in calibration model $2:$ minimum $=14.85 \mathrm{~g}$; maximum = $18.14 \mathrm{~g} ;$ mean $=$ $16.90 \mathrm{~g} ; \mathrm{SD}=1.19 \mathrm{~g}$. Protein statistics in prediction model 2 : minimum = $14.76 \mathrm{~g}$; maximum = $18.14 \mathrm{~g}$; mean $=16.92 \mathrm{~g} ; \mathrm{SD}=1.18 \mathrm{~g} . \mathrm{R}_{\mathrm{c}}^{2}=$ coefficient of determination for calibration; $\mathrm{R}_{\mathrm{p}}^{2}=$ coefficient of determination for prediction; RMSEC = root mean square error of calibration; RMSEP = root mean square error of prediction; $\mathrm{RPD}$ = residual predictive deviation; LS-SVM = least squares support vector machine; ICA-LS-SVM = independent component analysis-least squares support vector machine.
}

brands 6 and 7 were mixed. However, brands 1 and 7 could be identified by protein content and brands 4,5 , and 6 were identified by fat content. Therefore, the analysis of different components of the milk powders supported the view that the different brands used here could be identified well according to their different component contents. Contents of the 3 main components of brands 6 and 7 were close, whereas the brand identification of these 2 brands was good. Thus, short-wave NIRS was sensitive enough for brand identification. We concluded that because the contents of the milk powders were different between each 2 brands, in this study, 7 typical brands of milk powder were discriminated well by using the short-wave NIRS technique.

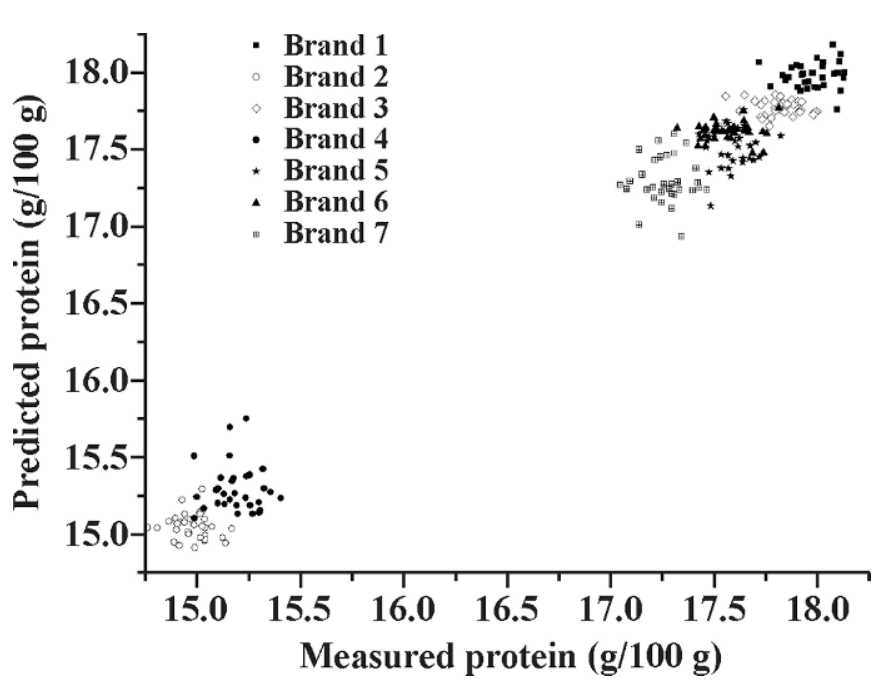

Figure 6. Correlation between predicted and measured values for protein content for the prediction set obtained by the least squares support vector machine process.

\section{CONCLUSIONS}

The present study provided insight into the shortwave NIR region, which was newly applied in the emerging field of milk powder analysis. We concluded that the short-wave NIRS technique is promising for conducting brand identification and content determination of the main components of milk powder. The chemometric models gave reliable results, suggesting the application value of short-wave NIRS. The operation of spectral measurement was simple and was more convenient than chemistry methods. More effort needs to be made to expand both the number and variety of samples, and sensitive wavelengths representing the main

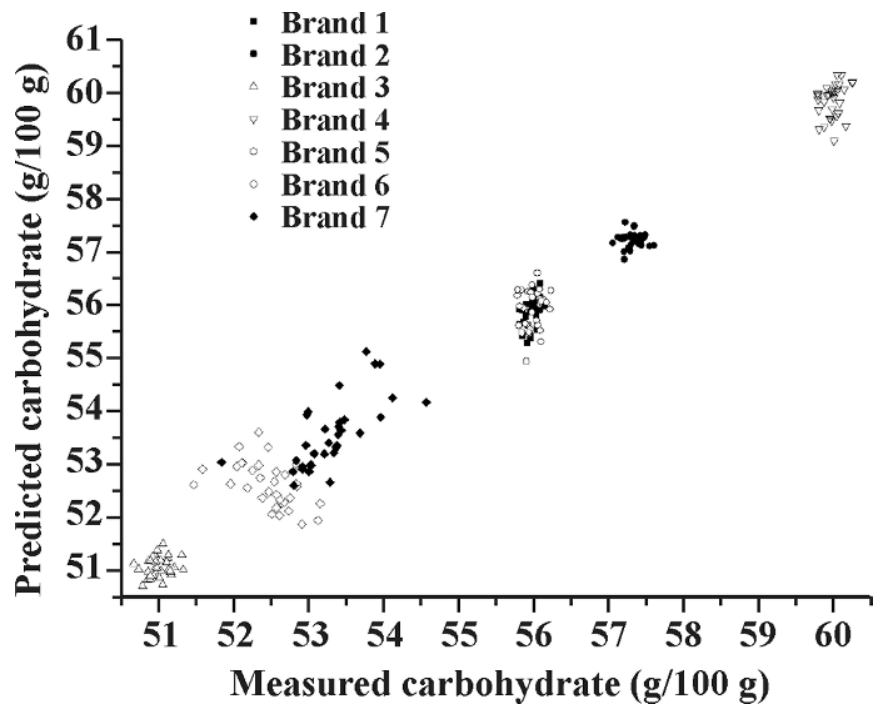

Figure 7. Correlation between predicted and measured values for carbohydrate content for the prediction set obtained by the least squares support vector machine process. 
BRAND AND COMPONENT ANALYSIS OF MILK POWDER BY INFRARED SPECTROSCOPY

Table 9. Results for carbohydrate determination in milk powder from the short-wave near-infrared spectra ${ }^{1}$

\begin{tabular}{lcccccccr}
\hline \multirow{2}{*}{ Set } & Model & \multicolumn{1}{c}{ Method } & $\mathrm{R}_{\mathrm{c}}^{2}$ or R $\mathrm{R}_{\mathrm{P}}^{2}$ & or RMSEP & Bias & Slope & Offset & RPD \\
\hline Calibration & \multirow{2}{*}{1} & LS-SVM & 1.000 & 0.014 & $-4.58 \mathrm{E}-05$ & 0.999 & 0.023 & 212.19 \\
& & ICA-LS-SVM & 0.990 & 0.277 & $-3.20 \mathrm{E}-09$ & 0.986 & 0.761 & 10.46 \\
& 2 & LS-SVM & 0.999 & 0.022 & $-1.62 \mathrm{E}-05$ & 0.999 & 0.034 & 129.96 \\
\multirow{3}{*}{ Prediction } & \multirow{2}{*}{1} & ICA-LS-SVM & 0.992 & 0.256 & $-2.70 \mathrm{E}-10$ & 0.987 & 0.713 & 11.34 \\
& & LS-SVM & 0.981 & 0.380 & 0.02 & 0.986 & 0.740 & \\
& \multirow{2}{*}{2} & ICA-LS-SVM & 0.980 & 0.408 & -0.02 & 0.979 & 1.103 & \\
& & LS-SVM & 0.984 & 0.369 & 0.04 & 0.965 & 1.934 & \\
\hline
\end{tabular}

${ }^{1}$ Carbohydrate statistics in calibration model 1 : minimum $=50.85 \mathrm{~g} ;$ maximum $=60.28 \mathrm{~g} ;$ mean $=55.22$ $\mathrm{g} ; \mathrm{SD}=2.89 \mathrm{~g}$. Carbohydrate statistics in prediction model $1:$ minimum $=50.66 \mathrm{~g}$; maximum $=60.20 \mathrm{~g}$; mean $=55.14 \mathrm{~g} ; \mathrm{SD}=2.91 \mathrm{~g}$. Carbohydrate statistics in calibration model $2:$ minimum $=50.83 \mathrm{~g} ;$ maximum $=$ $60.20 \mathrm{~g}$; mean $=55.19 \mathrm{~g} ; \mathrm{SD}=2.89 \mathrm{~g}$. Carbohydrate statistics in prediction model 2: minimum = $50.66 \mathrm{~g}$; maximum $=60.28 \mathrm{~g} ;$ mean $=55.16 \mathrm{~g} ; \mathrm{SD}=2.91 \mathrm{~g} . \mathrm{R}_{\mathrm{c}}^{2}=$ coefficient of determination for calibration; $\mathrm{R}_{\mathrm{p}}^{2}=$ coefficient of determination for prediction; RMSEC = root mean square error of calibration; RMSEP $=$ root mean square error of prediction; RPD = residual predictive deviation; LS-SVM = least squares support vector machine; ICA-LS-SVM = independent component analysis-least squares support vector machine.

components need to be selected to design a short-wave NIR device for brand identification and component determination of milk powder.

\section{ACKNOWLEDGMENTS}

This study was supported by the National Science and Technology Support Program (2006BAD10A04, 2006BAD10A07; Beijing, China), the Natural Science Foundation of China (project nos. 30671213, 60605011; Beijing, China), the Specialized Research Fund for the Doctoral Program in Higher Education (project no. 20040335034; Beijing, China).

\section{REFERENCES}

AOAC. 1984. Official Methods of Analysis. S. Williams, ed. AOAC, Arlington, VA.

Barabássy, S. 2001. The application of near infrared spectroscopy (NIR) technique for non-destructive investigation of mixed milk powder products. Mljekarstvo 51:263-272.

Barnes, R., M. Dhanoa, and J. Lister. 1989. Standard normal variate transformation and detrending of near infrared diffuse reflectance spectra. Appl. Spectrosc. 43:772-777.

Bittner, A., R. Marbach, and H. M. Heise. 1995. Multivariate calibration for protein, cholesterol and triglycerides in human plasma using short-wave near infrared spectrometry. J. Mol. Struct. 349:341-344.

Borin, A., M. F. Ferrao, C. Mello, D. A. Maretto, and R. J. Poppi. 2006. Least-squares support vector machines and near infrared spectroscopy for quantification of common adulterants in powdered milk. Anal. Chim. Acta 579:25-32.

Brás, L. P., S. A. Bernardino, J. A. Lopes, and J. C. Menezes. 2005. Multiblock PLS as an approach to compare and combine NIR and MIR spectra in calibrations of soybean flour. Chemom. Intell. Lab. Syst. 75:91-99.

Chen, Y. J., C. Iyo, and S. Kawano. 1999. Development of calibration with sample cell compensation for determining the fat content of unhomogenised raw milk by a simple near infrared transmittance method. J. Near Infrared Spectrosc. 7:265-273.
He, Y., D. Wu, and S. Feng. 2007. Fast measurement of sugar content of yogurt using Vis/NIR-spectroscopy. Int. J. Food Prop. 10:1-7.

Hyvärinen, A., and E. Oja. 1997. A fast fixed-point algorithm for independent component analysis. Neural Comput. 9:1483-1492.

Hyvärinen, A., and E. Oja. 2000. Independent component analysis: Algorithms and applications. Neural Netw. 13:411-430.

Li, J. Z., H. X. Liu, X. J. Yao, M. C. Liu, Z. D. Hu, and B. T. Fan. 2007. Structure-activity relationship study of oxindole-based inhibitors of cyclin-dependent kinases based on least-squares support vector machines. Anal. Chim. Acta 581:333-342.

Osborne, B. G., and T. Fearn. 1986. Near Infrared Spectroscopy in Food Analysis. Longman Scientific and Technical, Essex, UK.

Reeves, J. B., III. 1994. Effects of water on the spectra of model compounds. J. Near Infrared Spectrosc. 2:199-212.

Reid, L. M., T. Woodcock, C. P. O'Donnell, J. D. Kelly, and G. Downey. 2005. Differentiation of apple juice samples on the basis of heat treatment and variety using chemometric analysis of MIR and NIR data. Food Res. Int. 38:1109-1115.

Sasic, S., and Y. Ozaki. 2001. Short-wave near-infrared spectroscopy of biological fluids. 1. Quantitative analysis of fat, protein, and lactose in raw milk by partial least-squares regression and band assignment. Anal. Chem. 73:64-71.

Singer, A. 2006. Spectral independent component analysis. Appl. Comput. Harmon. Anal. 21:135-144.

Subedi, P. P., K. B. Walsh, and G. Owens. 2007. Prediction of mango eating quality at harvest using short-wave near infrared spectrometry. Postharvest Biol. Technol. 43:326-334.

Suykens, J. A. K., and J. Vanderwalle. 1999. Least squares support vector machine classifiers. Neural Process. Lett. 9:293-300.

Suykens, J. A. K., T. Van Gestel, J. De Brabanter, B. De Moor, and J. Vandewalle. 2002. Least Squares Support Vector Machines. World Scientific Publishing, Singapore.

Tsenkova, R., S. Atanassova, K. Toyoda, Y. Ozaki, K. Itoh, and T. Fearn. 1999. Near-infrared spectroscopy for dairy management: Measurement of unhomogenized milk composition. J. Dairy Sci. 82:2344-2351.

Vapnik, V. 1998. Statistical Learning Theory. John Wiley and Sons Inc., New York, NY.

Williams, P., and K. Norris. 2001. Near-infrared Technology in the Agricultural and Food Industries. 2nd ed. Am. Assoc. Cereal Chem., St. Paul, MN.

Wu, D., S. Feng, and Y. He. 2007. Infrared spectroscopy technique for the nondestructive measurement of fat content in milk powder. J. Dairy Sci. 90:3613-3619.

Wu, D., Y. He, S. Feng, and D.-W. Sun. 2008. Study on infrared spectroscopy technique for fast measurement of protein content in milk powder based on LS-SVM. J. Food Eng. 84:124-131. 